P0-0114 REPEATED HIGH-DOSE GAMMA GLOBULIN ADMINISTRATION WAS EFFECTIVE FOR GIANT CELL HEPATITIS WITH AUTOIMMUNE HEMOLYTIC ANEMMIA IN EARLY CHILDHOOD

T Endo, K Ito, T Sugiura, M Kamei, Y Ito. Pediatrics, Nagoya City University Graduate School of Medical Sciences, Nagoya, Japan

10.1136/archdischild-2014-307384.783

Background and aims There is no established treatment method for giant cell hepatitis with autoimmune hemolytic anaemia (GCH-AIHA) in early childhood. This study was designed to investigate whether repeated high-dose gamma globulin administration was effective for a case of GCH-AIHA in early childhood.

Methods The subject was a 10-month-old girl. At 6 months of age, examination for poor activity levels revealed that she was anaemic with elevated transaminase levels. She was then hospitalised at another hospital. Bone marrow testing ruled out the presence of any malignant diseases. She received blood transfusion and was administered steroids, but her condition did not improve; thus, she was transferred and hospitalised at our hospital. Upon admission, her liver was palpable at two finger breadths and her spleen was palpable at one finger breadth. Blood test results were as follows: haemoglobin, $7.0 \mathrm{~g} / \mathrm{dL}$; alanine transaminase, $354 \mathrm{U} / \mathrm{L}$; total bilirubin, $15.0 \mathrm{mg} / \mathrm{dL}$; direct bilirubin, $9.7 \mathrm{mg} / \mathrm{dL}$; direct and indirect Coombs' tests, positive and antinuclear antibodies, below 40×; and anti-liver-kidney microsomal-1 antibodies, negative. Liver biopsy confirmed giant cell hepatitis. Mild inflammatory cell infiltration was observed, although fibrosis was extremely mild. Considering her clinical course, the patient was diagnosed with GCH-AIHA. After steroid pulse therapy, administration of prednisolone and cyclosporine was continued but transaminase levels did not improve. Therefore, high-dose $(2 \mathrm{~g} / \mathrm{kg})$ gamma globulin was administered once per month for a total of four times.

Results The patient's anaemia improved and her transaminase levels dropped.

Conclusions Repeated high-dose gamma globulin administration is effective for GCH-AIHA.

\section{PO-0115 WITHDRAWN}

\section{PO-0116 RESULTS OF A SINGLE CENTRE REGISTER FOR PAEDIATRIC INTESTINAL FAILURE}

${ }^{1}$ D Hermans, ${ }^{2}$ S Scheers, ${ }^{1} \mathrm{~L}$ Bessalah, ${ }^{3} \mathrm{~F}$ Fusaro. ${ }^{1}$ Pediatrics, Cliniques Universitaires Saint Luc, Brussels, Belgium; '2Pediatric Gastroenterology Hepatology and Nutrition Unit, Cliniques Universitaires Saint Luc, Brussels, Belgium; ${ }^{3}$ Neonatal Surgery Unit, Children's Hospital Bambino Gesu, Roma, Italy

\subsection{6/archdischild-2014-307384.784}

Aim Intestinal failure (IF) is a rare condition requiring long term parenteral nutrition (PN). The complex management of IF needs a multidisciplinary approach by expert team of paediatricians, surgeons, gastroenterologists, nurses and dieticians. The risks of medical, surgical and nutritional complications must be prevented or managed appropriately. The primary aims of the register were to list the patients, the aetiology of IF, the therapeutic approach of the disease and the follow-up.
Methods We collected retrospectively the data of 21 patients followed in our centre for IF between 1999 and 2013.

Results Twenty-one patients were included, their age ranged between 2 mo and 15 yrs (mean 7.4 yrs). The causes of IF were short bowel syndrome (16), malabsorption (1) and intestinal motility disorders (4). Sex ratio was 0.29 (F/M). All children required home $\mathrm{PN}$. The duration of $\mathrm{PN}$ was $(43.1 \pm 46.9 \mathrm{mo})$. $10 / 21(47.6 \%)$ patients could be totally weaned from PN after $25.4 \pm 33.5$ months, 5/21 (24\%-after $109 \pm 74.9$ months) were off PN after longitudinal intestinal lengthening tapering (4LILT) or 1 after combined serial tapering enteroplasty (STEP) and (LILT). PN partially weaned in the 6/21 (23.8\%) remaining patients.

Conclusions Results of this single centre experience demonstrate that with a proactive multidisciplinary IF management, including surgical rehabilitation procedures, the majority of patients have obtained partial or full enteral autonomy. Extension of this register to other centre in Belgium or Europe would be interesting and probably enhance the quality of patient follow-up.

\section{PO-0117 CLINICAL AND EPIDEMIOLOGICAL CHARACTERISTICS OF COLIC IN THE INFANTS}

OM Horlenko, UG Dubinina, AO Yankovsyka, BM Halay, OA Pushkarenko, GB Cossey, VL Piridi. Pediatric with Infectiuos Diseases, Uzhgorod National University Medical Faculty, Uzhgorod, Ukraine

\subsection{6/archdischild-2014-307384.785}

Background and aims Intestinal colic in the infants is often found in early childhood and is one of the most frequent causes of complaints of parents to the paediatrician. They have functional genesis in $90 \%$ of cases, and terminated without treatment at the age of 3-4 months. Problem of Colic needs further study of the causes of disease and the possibility of its correction.

Methods We inspected of 66 children (aged from 6 to 66 days, $\mathrm{M} \pm \mathrm{m}, 33,52 \pm 3,85$ days) which have primary diagnosis colic on the bases of Clinical Children Hospital, uzhgorod, Ukraine.

Results The investigation contingent have follows assotiation diagnosis: Acute Respiratory Viral Infection (18,18 \pm 4,78\%), Pyelonephritis (6,06 $\pm 2,46 \%)$, Pneumonia (9,09 $\pm 3,57 \%)$ Syndrome increased neur-reflex excitability $(10,61 \pm 3,82 \%)$, Protein-energy malnutrition (13,64 $\pm 4,26 \%)$, Atopic dermatitis $(9,09 \pm 3,57 \%)$, Matabolic syndrome (3,03 $\pm 2,13 \%)$, Anaemia $(3,03 \pm 2,13 \%)$, Bullous dermatitis (4,55 $\pm 2,58 \%)$, Jaundice $(4,55 \pm 2,58)$, Torticollis $(3,03 \pm 2,13 \%)$, Perinatal infection $(3,03 \pm 2,13 \%)$. Body weight at the birth was $3214,50 \pm 84,57$ $\mathrm{h}$, at the time of inspection - 3785,24 $\pm 151,94 \mathrm{~h},(\mathrm{n}=66)$. Clinical signs in the children: Anxiety (100\%), Sharp cry $(6,06$ $\pm 2,46 \%)$, Weakness $(10,61 \pm 3,82 \%)$, Frequent regurgitation $(89,39 \pm 3,82 \%)$, Periodical Vomiting $(6,06 \pm 2,46)$, Flatulence (100), High. Temperature $(3,03 \pm 2,13)$ Vomiting (4,55 \pm $2,58)$, The rejection of breastfeeding $(3,03 \pm 2,13)$, Obstacle breathing $(18,18 \pm 4,78)$, Diarrhoea $(3,03 \pm 2,13)$.

Conclusions The colic diagnosed in the children with assotiation pathology-acute respiratory viral infection, syndrome increased neur-reflex excitability, protein-energy malnutrition. Intestinal colic were characterised by of Anxiety, Flatulence, Frequent regurgitation, Weakness, Obstacle breathing for our dates. The dominant factors in the development of colic are inflammation and nutritional desorder in the clinical design. 


\section{PO-0118 WITHDRAWN}

\section{PO-0119 ETIOLOGICAL DIAGNOSIS POSSIBILITIES IN ACUTE DIARRHOEA IN CHILDREN}

${ }^{1} \mathrm{~S}$ lurian, ${ }^{2} \mathrm{SI}$ lurian, ${ }^{2} \mathrm{C}$ Cazan. ${ }^{1} \mathrm{Clinical}$ Laboratory, Pediatric Hospital, Sibiu, Romania;

${ }^{2}$ Pediatric Clinic, "Lucian Blaga" University Pediatric Hospital, Sibiu, Romania

10.1136/archdischild-2014-307384.786

Background Acute diarrhoea (AD) plays an important role in paediatric patients because of its high incidence, severe clinical outcomes (dehydration) and epidemiological features (gastroenteritis spreads easily in children communities).

Aims To evaluate the aetiology of AD and epidemiological characteristics for hospitalised children during January 2013-January 2014.

Methods During mention period, there were performed stool cultures and Rotavirus/Adenovirus antigen testing from stool (immunochromatographic test) for patients admitted in paediatric hospital with $\mathrm{AD}$ diagnosis. The growth medium used for stool cultures: Hectoen-enteric and Selenite broth (for Salmonella, Shigella), Sorbitol MacConkey medium (for enteropathogen E. Coli -EPEC), CIN (for Yersinia) and CCDA (for Campylobacter, stool samples testing was initiated in July 2013). Exclusion criteria: positive stool culture for previously treated children.

Results There were performed 2138 stool cultures and 1358 tests for Rotavirus/Adenovirus. The authors have identified 73 Salmonella spp. cases, 12 Shigella spp. cases, 4 EPEC cases, 31 children with Campylobacter spp., 222 Rotavirus cases and 22 patients with positive test for Adenovirus. Authors remarked high incidence Salmonella infection in July -September period (39 cases, 53.4\%) and increased incidence of Rotavirus infection with 2 peaks totaling $52.7 \%$ of cases (January -December and May respectively).

Conclusions 1. Acute gastroenteritis major aetiology was the viral one; 2. Concerning bacterial aetiology of $\mathrm{AD}$, Campylobacter spp. represented the second cause (Salmonella spp. was more frequent); 3 . The aetiology awareness is useful in order to treat optimally patients with $\mathrm{AD} ; 4$. The antibiotics are recommended just for selected cases.

\section{PO-0120 WITHDRAWN}

\section{PO-0121 THE COMPOSITION OF PRETERM HUMAN MILK AND INFLUENCING FACTORS ON PROTEIN CONTENT}

A Kreissl, V Zwiauer, A Repa, C Binder, M Thanhaeuser, A Berger, N Haiden. Department of Pediatrics Division of Neonatology Pediatric Intensive Care Medicine and Neuropediatrics, Medical University of Vienna, Vienna, Austria

\subsection{6/archdischild-2014-307384.787}

Background Human milk (HM) is unique and the gold standard for feeding preterm infants. However, HM contains insufficient quantity of energy, calcium, phosphorus and protein - the key nutrient - for preterm infants. Low protein intake is associated with poor weight gain, length and neurocognitive outcomes. Therefore, human milk fortifiers have been developed. The aim of Compo-HMA study was to measure the composition and variation in preterm HM, the impact of lactation period and different milk processing types on the nutritional content with MIRIS Human Milk Analyzer (HMA).

Methods HM was taken from mothers of premature infants born with a birthweight $<1500 \mathrm{~g}$ and a gestational age $<32$ weeks. Protein, fat, lactose and energy composition was measured with MIRIS HMA subjected to different milk processing types fresh/fresh pasteurised/thawed/thawed pasteurised. Furthermore, the relation between protein content and lactation days has been evaluated.

Results Native HM $(\mathrm{n}=83)$ contained in mean $1.1 \pm 0.37 \mathrm{~g}$ protein, $3.2 \pm 0.85 \mathrm{~g}$ fat, $6.6 \pm 0.34 \mathrm{~g}$ lactose and $59.8 \pm$ $10.65 \mathrm{kcal}$ energy $/ 100 \mathrm{ml}$ HM. Maximum variation was observed in protein content with $0.2-2.2 \mathrm{~g} / 100 \mathrm{ml} \mathrm{HM}$. Lactation day correlated significantly with protein content $(\mathrm{p}<$ $0.0001 ; \mathrm{r}=-, 4243)$. Processing of HM had no effects on protein-or nutritional level.

Conclusions Variations in macronutrients from mother to mother are high, particularly in protein. Protein significantly decreased with lactation period. Various conditions of HM processing had no effect on measured nutritional content. MIRIS is an adjuvant tool to provide actual nutrient composition of HM. The results indicate that HM analysis is important for individualised fortification and improves the nutritional management of premature infants.

\section{PO-0122 INCIDENCE AND RISK FACTORS FOR THIAMINE DEFICIENCY DURING A STAY IN A PAEDIATRIC INTENSIVE CARE UNIT}

LF Lima, H Leite. Pediatrics, Federal University of Sao Paulo, Sao Paulo, Brazil

\subsection{6/archdischild-2014-307384.788}

Background Thiamine deficiency (TD) has been reported in critically ill patients, both adult and paediatric. The risk factors and the outcomes associated with the development of TD in intensive care unit patients are not established. We aimed to determine the incidence and the risk factors associated with TD in children during the first 10 days of stay in a paediatric intensive care unit (PICU).

Methods In a prospective cohort study, 202 children admitted to PICU had thiamine whole blood concentrations monitored on the first, 5th and 10th day of PICU stay. Age, thiamine intake, C-Reactive Protein, malnutrition (WHO growth standards), admission diagnosis, disease severity Paediatric Index of Mortality 2 (PIM2), furosemide and dialysis use were analysed as risk factors for TD. An HPLC-based method (high performance liquid chromatography) was used to measure whole-blood thiamin concentrations. The data were analysed using a Binomial Generalised Estimating Equations model, which includes the correlation between admission, 5th and 10th day responses.

Results TD incidence rate was $15 \%$ during the first 10 days of ICU stay; 56 cases $(28 \%)$ cases were detected on admission and 4 new cases occurred during the follow up. Decreased thiamine concentrations were associated with C-Reactive Protein increase during the PICU stay (OR: -0.035 ; 95\% CI: $-0.06-0.008 ; \mathrm{p}=$ 0.01 ), but not with malnutrition $(\mathrm{p}=0.09)$.

Conclusions 1) TD incidence is frequent on admission and decreases during the ICU stay; 2) Systemic inflammation is associated with decreased thiamine concentrations upon admission and during the follow up. 